\title{
Maintenance Chemotherapy in Ovarian Cancer: A Trial-Sequential Analysis
}

\author{
Andrea Messori*, Valeria Fadda, Dario Maratea, Sabrina Trippoli \\ HTA Unit, ESTAV Toscana Centro, Regional Health Service, Firenze, Italy. \\ Email: "andrea.messori@estav-centro.toscana.it
}

Received July $26^{\text {th }}, 2013$; revised August $21^{\text {st }}, 2013$; accepted August $26^{\text {th }}, 2013$

Copyright (C) 2013 Andrea Messori et al. This is an open access article distributed under the Creative Commons Attribution License, which permits unrestricted use, distribution, and reproduction in any medium, provided the original work is properly cited.

\begin{abstract}
In patients with epithelian ovarian cancer who have achieved remission after initial surgery and induction chemotherapy, the role of maintenance chemotherapy is controversial. We carried out a trial-sequential analysis that included 4 randomised controlled trials. The end-point was progression at 3 years while the boundary for non-inferiority was set at $\pm 20 \%$ in risk ratio. The results of our trial-sequential analysis indicated the futility of maintenance chemotherapy, i.e. proof of no effectiveness. Consequently, no further trials of this type should be performed to assess the effectiveness of this intervention in this clinical condition.
\end{abstract}

Keywords: Chemotherapy; Ovarian Cancer; Trial-Sequential Analysis; Futility

\section{Introduction}

In patients with epithelian ovarian cancer who have achieved remission after initial surgery and induction chemotherapy, the role of maintenance chemotherapy is controversial. This pharmacological intervention is aimed at prolonging the duration of remission and improving overall survival; however, conflicting results have been reported on its effectiveness in comparison with observation alone.

\section{Results and Discussion}

Mei and coworkers [1] have recently published a metaanalysis on this issue based on 4 randomised controlled trials (RCTs) that used different types of conventional cytotoxic chemotherapy (e.g. platinum agents, doxorubicin, topotecan or paclitaxel). No proof of effectiveness was found; the pooled probability of progression-free survival (PFS) for maintenance chemotherapy vs observation alone was $1.07(95 \%$ confidence interval $[\mathrm{CI}]$ : 0.91 to 1.25 ), which corresponds to a relative risk of progression of 0.93 (95\% CI: 0.80 to 1.10$)$. Hence, this meta-analysis concluded that maintenance chemotherapy was not superior to observation alone, i.e. no proof of effectiveness.

To better interpret the implications of this negative finding, we applied trial-sequential analysis (TSA) [2-5]

${ }^{*}$ Corresponding author. to this data-set of survival information in order to determine whether the data available on this treatment simply indicate no proof of effectiveness or conclusive futility (i.e. proof of no effectiveness).

Our TSA employed the following assumptions: type 1 error, $5 \%$ (two-sided); power, $80 \%$; event frequency for controls, $48 \%$ (i.e. the overall event rate found in the 4 control groups). The boundaries for concluding superiority or inferiority or futility were calculated according to the O'Brien-Fleming alpha-spending function. A specific statistical software was used (User Manual for TSA, Copenhagen Trial Unit 2011, see www.ctu.dk/tsa). As usual, the main result of TSA was expressed through the graph of cumulative z-curve. The distinction between inconclusiveness and futility is not assumption-free, but depends on the choice of a specific threshold for incremental clinical effectiveness. In our TSA, this threshold was set at a relative risk reduction (RRR) of $20 \%$; this value was derived from the outcomes reported in the "most positive" trials evaluating remission rates and/or progression [1].

Figure 1 shows the results of our TSA in which "events" were cases of progression at 3 years. The raw data from the 4 trials are presented in the supplementary web material. According to our pre-determined assumptions, the TSA of these data indicated the futility of maintenance chemotherapy, i.e. proof of no effectiveness. Interestingly enough, although the optimal information 


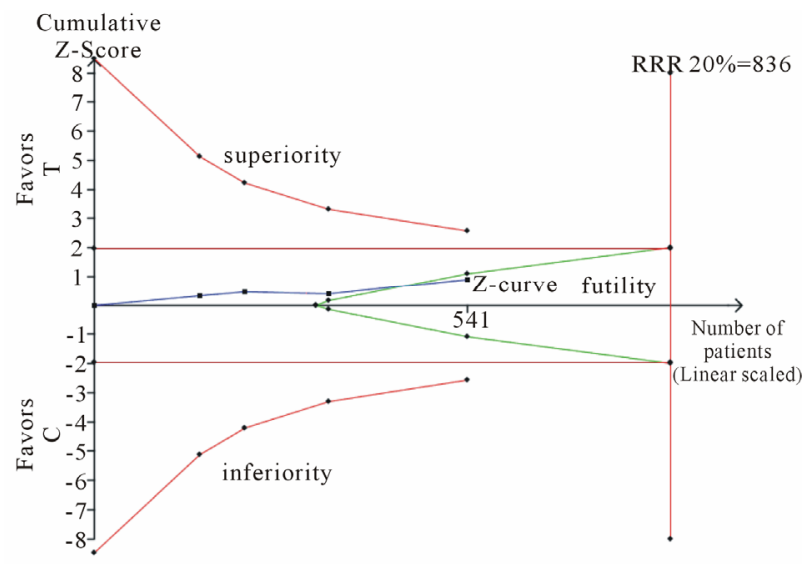

Figure 1. Maintenance chemotherapy versus observation in ovarian cancer: trial sequential analysis of 4 randomized trials (end-point $=$ progression). The z-curve (in blue) is composed of consecutive segments that correspond to individual trials; trials are plotted in chronological order (from left to right). The $x$-axis indicates the cumulative number of patients. At the cumulative number of 541 included patients, the curve has crossed the boundaries of futility. The optimal information size is 836 patients. Abbreviations and symbols: red lines are the boundaries for superiority or inferiority; green lines are the boundaries for futility. $\mathrm{T}$, treatment group; $\mathrm{C}$, control group.

size was estimated at a cumulative number of 836 patients, the 4 trials involving a total of 514 patients were already sufficient to prove futility.

The conclusion of our analysis depends on whether or not the threshold of incremental effectiveness (i.e. RRR $=20 \%$ ) is agreed upon or, in other words, if this threshold is assumed to reasonably reflect the criterion of clinical relevance. One should keep in mind that, in disease conditions in which life expectancy is relatively short, a RRR of $20 \%$ generally translates into an absolute survival improvement of only a few months; hence, if one considers that median PFS in these patients is slightly more than 24 months [6], a relative improvement of $20 \%$ corresponds to an absolute improvement in median PFS of less than 5 months, which can be considered an acceptable threshold in terms of clinical relevance.

\section{Conclusion}

In conclusion, if our assumptions are accepted, the results of our analysis can be seen as the proof of no effectiveness of maintenance chemotherapy in ovarian cancer based on conventional cytotoxic agents. Consequently, no further trials of this type should be performed to assess the effectiveness of this intervention in this clinical condition.

\section{REFERENCES}

[1] L. Mei, H. Chen, D. M. Wei, F. Fang, G. J. Liu, H. Y. Xie, et al., "Maintenance Chemotherapy for Ovarian Cancer," Cochrane Database Systematic Reviews, Vol. 6, 2013, Article ID: CD007414. doi:10.1002/14651858.CD007414.pub3

[2] J. Wetterslev, K. Thorlund, J. Brok and C. Gluud, "Trial Sequential Analysis May Establish When Firm Evidence Is Reached in Cumulative Meta-Analysis," Journal of Clinical Epidemiology, Vol. 61, No. 1, 2008, pp. 64-75. doi:10.1016/j.jclinepi.2007.03.013

[3] J. Brok, K. Thorlund, C. Gluud and J. Wetterslev, "Trial Sequential Analysis Reveals Insufficient Information Size and Potentially False Positive Results in Many MetaAnalyses," Journal of Clinical Epidemiology, Vol. 61, No. 8, 2008, pp. 763-769. doi:10.1016/j.jclinepi.2007.10.007

[4] A. Messori, V. Fadda, D. Maratea and S. Trippoli, “ $\omega-3$ Fatty Acid Supplements Form Secondary Prevention of Cardiovascular Disease: From No Proof of Effectiveness to Proof of No Effectiveness," JAMA Internal Medicine, 2013, Epub.

[5] D. Maratea, V. Fadda, S. Trippoli and A. Messori, "OffPump versus On-Pump Coronary Artery Bypass Grafting: Quantifying Information Size by Trial Sequential Analysis," European Journal of Internal Medicine, 2013, Epub.

[6] S. Pecorelli, G. Favalli, A. Gadducci, D. Katsaros, P. B. Panici, A. Carpi, et al., "Phase III Trial of Observation versus Six Courses of Paclitaxel in Patients with Advanced Epithelial Ovarian Cancer in Complete Response after Six Courses of Paclitaxel/Platinum-Based Chemotherapy: Final Results of the After-6 Protocol 1," Journal of Clinical Oncology, Vol. 27, No. 28, 2009, pp. 46424648. doi:10.1200/JCO.2009.21.9691 